\title{
Direct Probing of the Mott Crossover in the SU(N) Fermi-Hubbard Model
}

\author{
Christian Hofrichter, Luis Riegger, Francesco Scazza, Moritz Höfer, Diogo Rio Fernandes, \\ Immanuel Bloch, and Simon Fölling \\ Ludwig-Maximilians-Universität, Schellingstraße 4, 80799 München, Germany \\ and Max-Planck-Institut für Quantenoptik, Hans-Kopfermann-Straße 1, 85748 Garching, Germany
}

(Received 11 December 2015; published 1 June 2016)

\begin{abstract}
We report on a detailed experimental investigation of the equation of state (EoS) of the three-dimensional Fermi-Hubbard model (FHM) in its generalized $\mathrm{SU}(N)$-symmetric form, using a degenerate ytterbium gas in an optical lattice. In its more common spin-1/2 form, the FHM is a central model of condensed-matter physics. The generalization to $N>2$ was first used to describe multi-orbital materials and is expected to exhibit novel many-body phases in a complex phase diagram. By realizing and locally probing the $\mathrm{SU}(N)$ FHM with ultracold atoms, we obtain model-free access to thermodynamic quantities. The measurement of the EoS and the local compressibility allows us to characterize the crossover from a compressible metal to an incompressible Mott insulator. We reach specific entropies above Néel order but below that of uncorrelated spins. Having access to the EoS of such a system represents an important step towards probing predicted novel $\mathrm{SU}(N)$ phases.
\end{abstract}

DOI: 10.1103/PhysRevX.6.021030

Subject Areas: Atomic and Molecular Physics, Condensed Matter Physics, Quantum Physics

Strongly correlated fermionic many-body systems play a fundamental role in modern condensed-matter physics. A central model for these systems is the Fermi-Hubbard model (FHM), originally developed for describing interacting electrons in a crystal. It explains a wide range of observed phenomena such as metal-to-insulator transitions and magnetic order, and is believed to capture the essential physics of $d$-wave superfluidity in high-temperature superconductors [1,2]. The generalized $\mathrm{SU}(N)$-symmetric version of the FHM was originally studied in the context of transition-metal oxides with effective higher spin [3].

Although the FHM has been the object of a large number of studies in past decades, reaching a complete understanding has remained an elusive task, even for the spin$1 / 2$ case. For strong repulsive interactions, the SU(2) FHM is known to give rise to a paramagnetic Mott insulator, where antiferromagnetic order emerges below the Néel temperature. Already in this limit of strong interactions and low temperature, the $\mathrm{SU}(N>2)$ case has been predicted to exhibit a rich phase diagram with a variety of different correlated states [4-13].

The development of experimental implementations of the three-dimensional (3D) FHM with ultracold atoms has provided a new approach for advancing our understanding of strongly correlated fermions in lattices [14]. The recent realization of degenerate gases of strontium and ytterbium $[15,16]$, in combination with optical lattices, allows us to

Published by the American Physical Society under the terms of the Creative Commons Attribution 3.0 License. Further distribution of this work must maintain attribution to the author(s) and the published article's title, journal citation, and DOI. extend this beyond the conventional spin- $1 / 2$ FHM and to access the more general $\mathrm{SU}(N>2)$ symmetry. Numerical calculations in this regime are, so far, mostly limited to $T=0$, low dimensions, or approximated correlations. Accurate predictions of thermodynamic quantities are even harder to obtain than for the SU(2) case, as most algorithms struggle with larger $N$ due to the unfavorable scaling of the Hilbert space. Probing the thermodynamics of such systems, implemented with ultracold atoms, provides access to this challenging regime and allows for the testing of novel algorithms by direct comparison of thermodynamical quantities.

Fermionic ${ }^{173} \mathrm{Yb}$ has nuclear spin $I=5 / 2$ but no electronic angular momentum in the ground state $(J=0)$. Therefore, nuclear and electronic angular momenta are decoupled, making the atomic interactions independent of the nuclear spin state and making the system $\mathrm{SU}(N)$ symmetric, with $N \leq 2 I+1=6$ being the number of populated nuclear spin states [17]. One important manifestation of large $N$ is an enhanced Pomeranchuk effect, leading to a suppression of particle-hole excitations in the lattice [18]. Owing to this, the first evidence of an incompressible phase in an $\mathrm{SU}(6){ }^{173} \mathrm{Yb}$ gas has recently been reported, at an entropy level that does not support a Mott insulator in lower spin gases [19].

The $\mathrm{SU}(N)$-symmetric FHM for arbitrary spin multiplicity $N$ can be written as [11]

$$
\hat{H}=-t \sum_{\langle i, j\rangle, \sigma}\left(\hat{c}_{i \sigma}^{\dagger} \hat{c}_{j \sigma}+\text { H.c. }\right)+\frac{U}{2} \sum_{i, \sigma \neq \sigma^{\prime}} \hat{n}_{i \sigma} \hat{n}_{i \sigma^{\prime}}+\sum_{i, \sigma} V_{i} \hat{n}_{i \sigma} .
$$

Here, $\langle i, j\rangle$ denote neighboring lattice site indices, $t$ is the tunneling matrix element between them, $U$ is the on-site 
interaction, and $V_{i}$ is a position-dependent energy offset that accounts for the confining potential. We denote with $t^{*}=12 t$ the kinetic energy associated with the bandwidth of the 3D lattice. The operator $\hat{c}_{i \sigma}$ annihilates a fermion at site $i$ with spin index $\sigma=1, \ldots, N$, and $\hat{n}_{i \sigma}=\hat{c}_{i \sigma}^{\dagger} \hat{c}_{i \sigma}$ are the respective number operators.

In this work, we measure and analyze the equation of state (EoS) of an $\mathrm{SU}(N)$ Fermi gas in a cubic optical lattice, for temperatures above the magnetic ordering temperature. The EoS is a thermodynamic relation that contains all the macroscopic properties of the system and, because of its generality, is particularly well suited to benchmark numerical simulations [20,21]. We take advantage of the confining potential and the local density approximation (LDA) to map the trapped heterogeneous gas with a fixed number of particles to a locally homogeneous gas in the grand canonical ensemble with $\mu_{i}=\mu_{0}-V_{i}$, where $\mu_{0}$ is the chemical potential at the center of the trap. The validity of the LDA for fermions trapped in optical lattices above the Néel temperature was previously verified by numerical calculations [22]. We determine the EoS for the density $n\left(\mu, T, N, U, t^{*}\right)$ over a wide range of parameters. In particular, we focus on the highest spin multiplicity of our system $N=6$ and on the case $N=3$, which was the subject of several theoretical studies [8-10,12,13,23]. By deriving the local compressibility directly from the measured EoS, we are able to detect the emergence of the incompressible Mott phase.

In our experiment, we start by preparing a degenerate Fermi gas of ${ }^{173} \mathrm{Yb}$ with initially $N=6$ equally populated spin components via evaporative cooling in a crossed optical dipole trap (see Appendix A for details). We then set $N$ by removing individual spin components. The Fermi gas is then loaded into the lowest energy band of a 3D optical lattice with cubic symmetry and lattice spacing $d=\lambda / 2$ operating at a wavelength of $\lambda=759 \mathrm{~nm}$ (see Appendix B). We vary the lattice depth between $3 E_{r}$ and $15 E_{r}$, with $E_{r}=h^{2} / 2 m \lambda^{2}$ being the recoil energy, a range for which the tight-binding approximation is valid. Adjusting the lattice depth allows us to tune the system from $U / t^{*}=0.128_{-0.008}^{+0.004}$ to $U / t^{*}=11.0_{-1.0}^{+1.1}$, spanning a range of 2 orders of magnitude.

In order to measure the local atomic density in the trap, we probe the cloud by performing in situ spin-independent absorption imaging along the $z$ axis of the lattice. The spatial resolution is approximately $1.2 \mu \mathrm{m} \approx 3.2 d$. Because of the high optical density of the trapped cloud, saturated absorption imaging at high light intensity is used (see Appendix C and Ref. [24] for details). The resulting integrated two-dimensional density distribution $\tilde{n}(x, y)$ is shown in Fig. 1(a). Exploiting the carefully characterized geometry of the trap configuration, we determine the local 3D density $n(r, y)$ by performing an inverse Abel transform with $y$ as the symmetry axis [25]. The elliptic trap symmetry around the $y$ axis is taken into account in the (a)
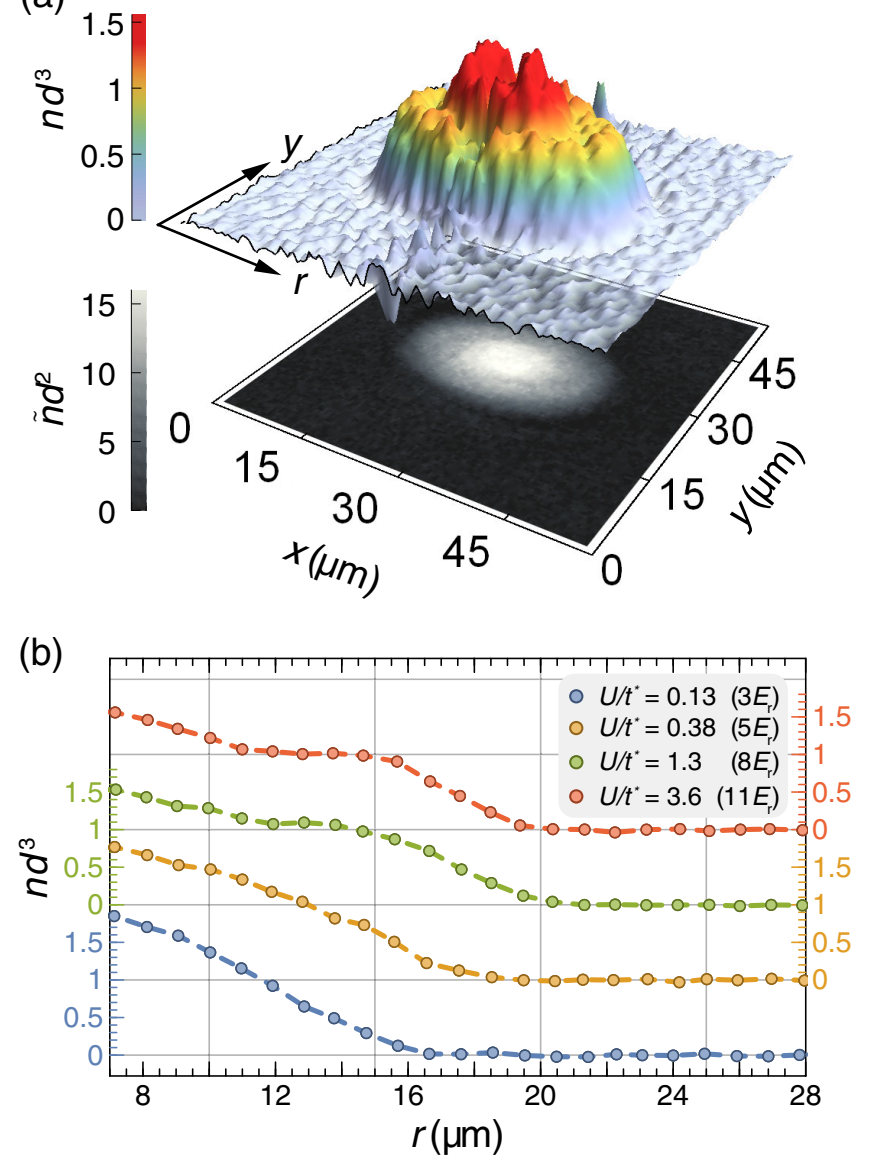

FIG. 1. Density profile of an SU(6) Fermi gas in an optical lattice with harmonic confinement. (a) Measured density distribution integrated along the line of sight (bottom) and local trap density obtained by performing the inverse Abel transform, displaying a plateau $n(r, y)=1$ (top) for interaction strength $U / t^{*}=6.4\left(V=13 E_{r}\right)$. Three experimental realizations are averaged for the displayed data. (b) Local atomic density as a function of the distance to the trap center for different interaction parameters $U / t^{*}$.

Abel transform by an appropriate rescaling along the $z$ direction. Since the transformation becomes very sensitive to noise close to the symmetry axis, we consider only data with $r>7 \mu \mathrm{m}$, where the standard error of the mean (s.e.m.) is typically $2 \%$ or lower. In Fig. 1(b), the azimuthally averaged density is shown as a function of the distance to the trap center for different values of the lattice depth. For the averaging, data within $\pm 4.8 \mu \mathrm{m}$ along the $y$ axis were used.

Two regimes can be distinguished: For low lattice depths, where the interaction energy is smaller than the kinetic energy $\left(U<t^{*}\right)$, the density decreases smoothly from the center to the edge of the trap, indicating that the system is compressible everywhere. For high lattice depths $\left(U>t^{*}\right)$, we observe the formation of a plateau of constant density, as expected for a Mott insulating state [26]. In this region, the atomic density computed using an independent 
calibration is compatible with the expected value of one fermion per lattice site (see Appendix E). Because of the higher precision of this measurement, the density of an $\mathrm{SU}(6)$ insulator in this region is used as the density reference in this paper.

To determine thermodynamic quantities, the EoS is obtained by relating the $3 \mathrm{D}$ atom density distribution and the chemical potential: $n\left[\mu=\mu_{0}-V(r, y)\right]$. This is done for different values of interaction strength $U / t^{*}$ and number of spin components $N=3$, 6. In Fig. 2, examples for small $\left(U / t^{*}=0.128\right)$, intermediate $\left(U / t^{*}=0.89\right)$, and large $\left(U / t^{*}=3.6\right)$ interaction strengths are given.

In the presence of a weak lattice $\left(U \ll t^{*}\right)$, the system is in a normal metallic state, well described by the $\mathrm{SU}(N)$ Fermiliquid theory [27] with enhanced mass and interactions due to the presence of the lattice. In particular, for low densities $n d^{3} \ll 1$, interactions can be neglected, and the EoS is well approximated by that of a noninteracting Fermi gas, as shown in Fig. 2(a). We use this observation to determine $\mu_{0}$ of the gases with $U / t^{*} \leq 0.89$ (see Appendix $\mathrm{D}$ for details). For high densities $n d^{3} \gtrsim 1$, the approximation fails. In this regime, the deviation from the noninteracting model increases as a function of $N$ because of the higher number of particles and interaction channels for a given value of $\mu$, scaling approximately as $(N-1)$ [27].

For deep lattice potentials, tunneling is highly suppressed $\left(t \ll k_{B} T\right)$ and interactions are strong $\left(U \gg t^{*}\right)$. In this regime, the lattice sites can approximately be regarded as independent. We construct a low-tunneling model using a high-temperature series expansion (HTSE) up to $\mathcal{O}\left(t / k_{B} T\right)^{2}[18,28]$ (see Appendix D for details). This model fits the measured EoS data well, with $T=$ $0.13(1) U / k_{B}$ and $T=0.18(1) U / k_{B}$ for $\mathrm{SU}(6)$ and $\mathrm{SU}(3)$, respectively [see Fig. 2(c)]. The lower temperature in the SU(6) case is due to both the lower initial temperature and the stronger Pomeranchuk effect. As the HTSE fit sensitivity to the trap confinement is higher than the precision of the independent trap calibration, we allow a variation of the confinement parameter in the model within the bounds given by the calibration.

The role of the $\mathrm{SU}(N)$ symmetry in the EoS is simple in the limit of negligible interactions: The density scales linearly with $N$ for fixed chemical potential. This dependence is well reproduced by the data, as shown in Fig. 2(a). For high lattice depths, and therefore larger $U / t^{*}$, the effect is twofold: First, the Pomeranchuk effect leads to lower temperatures in systems with higher $N[18,19]$. Second, for a given finite temperature, the EoS in the strongly interacting regime is $N$ dependent in a nontrivial way because of the different quantum statistics [12]. To illustrate this, we compare the low-tunneling model for the $\mathrm{SU}(2)$ case and the EoS for SU(6) in Fig. 2(c). As shown in the inset, a measurable discrepancy is present because of the different $\mathrm{N}$-dependent quantum statistics, which cannot be attributed to different temperature $T$ or central chemical potential $\mu_{0}$.
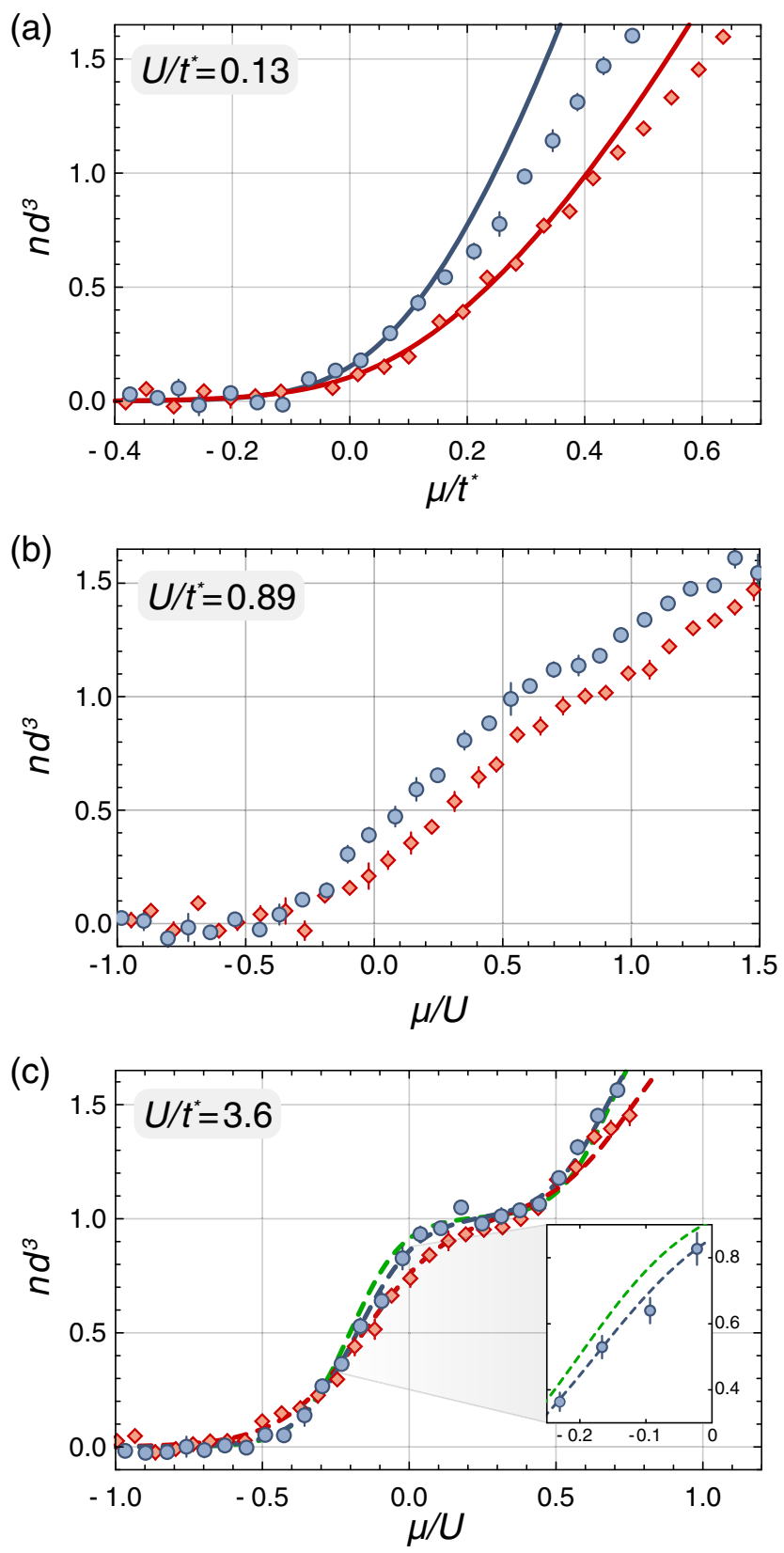

FIG. 2. EoS of the SU(6)- (blue circles) and the SU(3)- (red diamonds) spin symmetric Fermi gas in a lattice. Density as a function of the chemical potential for various interaction strengths: (a) $U / t^{*}=0.128$ (b) $U / t^{*}=0.89$ and (c) $U / t^{*}=3.6$. Solid lines are fits to the non-interacting Fermi gas EoS, with points $n d^{3}<0.5$ included in the fit. Dashed lines are the fits to the low tunneling model for $\mathrm{SU}(6)$ (blue) and $\mathrm{SU}(3)$ (red). An $\mathrm{SU}(2)$ model fit to the SU(6) data is shown for comparison (green), with the temperature $T$ and the central chemical potential $\mu_{0}$ as fit parameters. Error bars are the standard error of the mean (s.e.m. of the binned data).

In the regime of intermediate interaction strength, comparable to the kinetic energy $\left(U \approx t^{*}\right)$, the system is in a strongly correlated many-body state. No exact determination of the EoS is available yet to compare to our data, 


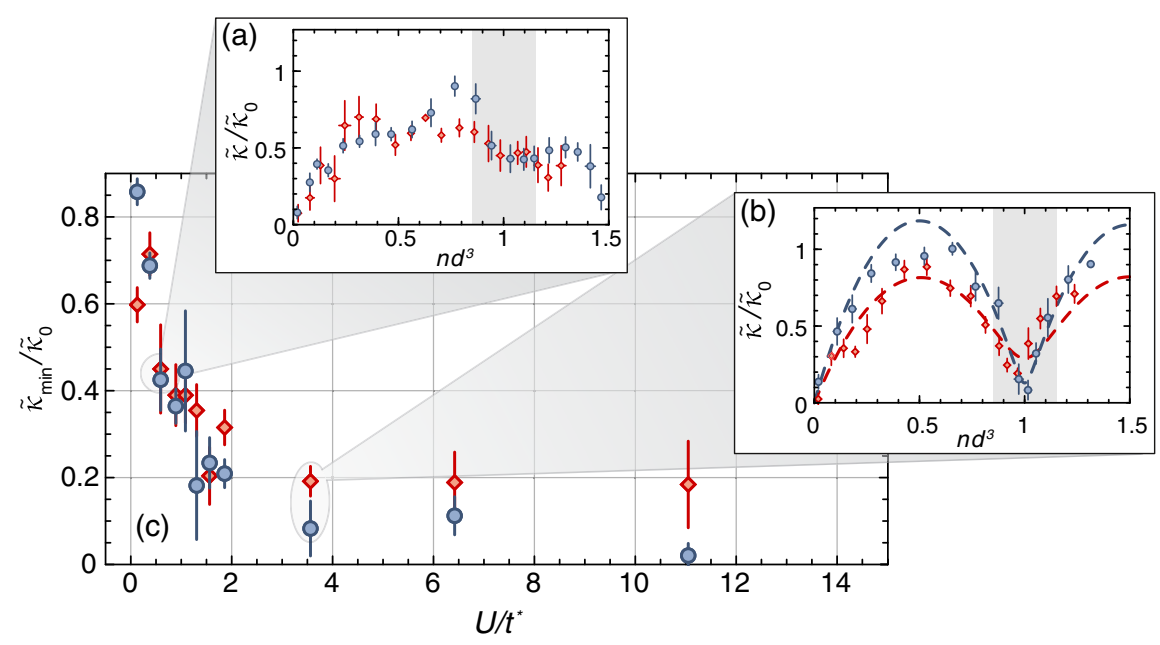

FIG. 3. Compressibility of SU(6)- (blue circles) and SU(3)- (red diamonds) spin-symmetric Fermi gases in a lattice. (a) compressibility as a function of density in the intermediate regime $U / t^{*}=0.59$. (b) compressibility as a function of density in the strongly interacting regime $U / t^{*}=3.6$. The dashed lines are obtained by deriving the fitted low-tunneling model plotted in Fig. 2(c). (c) minimal compressibility $\tilde{\kappa}_{\min } / \tilde{\kappa}_{0}$ as a function of interaction strength, where $\tilde{\kappa}_{0}$ is the compressibility of a non-interacting SU(6) Fermi gas at $T=0$ and $n=1 / d^{3}$. The minimum of the compressibility $\tilde{\kappa}(n)$ in an interval around unit filling (shaded area in insets) is used as $\tilde{\kappa}_{\text {min }}$. Error bars denote the confidence intervals of the linear regression used to obtain $\tilde{\kappa}$.

displayed in Fig. 2(b). Nevertheless, having model-free access to the EoS allows us to directly determine the local compressibility $\tilde{\kappa}=n^{2} \kappa=\partial n /\left.\partial \mu\right|_{T}$. This can be used to probe the emergence of the incompressible phase and to study the crossover between metal and Mott insulator, as shown in Fig. 3. For the strongly interacting case, we distinguish a metallic outer layer $0<n d^{3}<1$ and a metallic core $1<n d^{3}$, separated by a Mott shell with $n d^{3}=1$. In this regime, the low-tunneling model compares well with our results, with very small compressibilities in the Mott regions.

To characterize the onset of the Mott phase, we determine the minimum of compressibility $\tilde{\kappa}_{\min }$ around unit filling, in a range $n d^{3} \in[0.85,1.15]$, as a function of the interaction parameter (Fig. 3). We observe a suppression of $\tilde{\kappa}_{\text {min }}$ by roughly 1 order of magnitude when increasing the interaction strength. For large $U / t^{*}$, the compressibility saturates at a minimum value and the Mott shell is formed. The behavior is consistent with numerical calculations [23].

To further characterize the state in the lattice, we estimate the entropy per particle $s$ of the SU(6) gas. We determine the entropy before and after a round-trip, consisting of loading the atoms into the lattice and back into the dipole trap, by measuring the degeneracy parameter $T / T_{F}$ in the dipole trap, where $T_{F}$ is the Fermi temperature. This gives a lower and an upper estimate for $s$ in the lattice (see Fig. 4). For low lattice depths, we observe a constant entropy rise associated with the transfer from the bulk into the lattice and back. Above $U \approx t^{*}$, with the appearance of the incompressible phase, we find an additional entropy increase. This increase also persists for very low ramp speeds, which could indicate diminished adiabaticity due to the insulating phase crossover hindering mass flow. However, we observe that also for these high lattice depths, the entropy is at or below the maximum spin entropy $s / k_{B}=\ln (6)$ even after a full round-trip that includes the reversed second ramp. This indicates that the orientations of the spins in the lattice are likely not fully random,

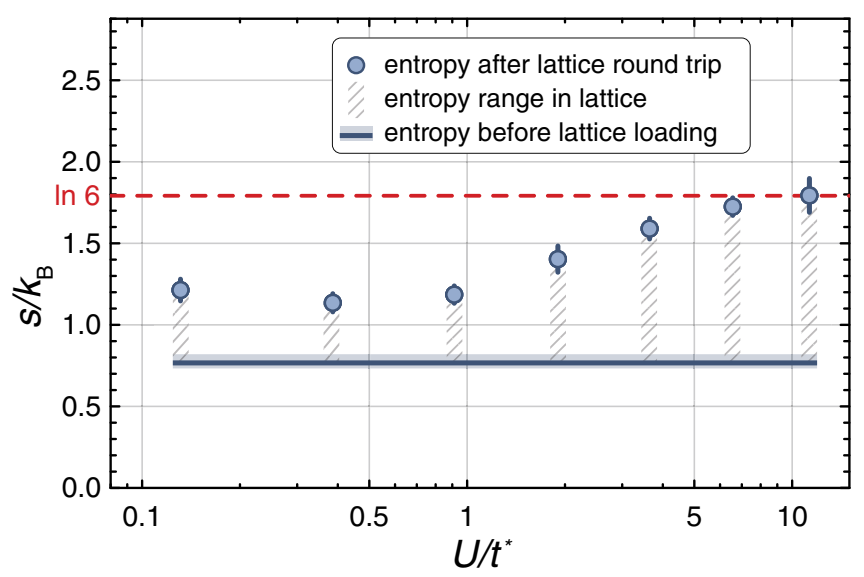

FIG. 4. Entropy per particle $s$ of the SU(6)-symmetric gas before and after the lattice round-trip sequence. The blue line represents the entropy measured in the bulk before transfer into the lattice. Blue points are measurements after round-trip loading into the lattice and back to the bulk. The entropy of the gas in the lattice is constrained to the region between these two measurements. The entropy of a system with our parameters and fully random spin orientations is close to $s / k_{B}=\ln 6$ (red line). Error bars and blue shaded region are the s.e.m. of the temperature measurements. 
allowing for the presence of partial spin correlations in the system.

In the regime of validity of the low-tunneling model, lower entropy corresponds to lower temperature and Mott shells with sharper edges. Although fitting the model provides a good match to the measured SU(6)-EoS data as shown in Fig. 2(c), the obtained temperature is significantly higher than that expected for the very low entropy measured in the round-trip experiment.

An interpretation could be that a suppression of mass flow close to the Mott crossover prevents the formation of sharp Mott shell edges, yielding higher fitted temperatures. This nonadiabatic freezing of the mass flow would then have to be largely reversible in order to explain the fact that the measured entropy in the equilibrated gas after the round-trip ramp is still lower than what the result of the HTSE fit inside the lattice suggests.

In the SU(3) case, the same effect is not observed. However, here the measured entropies are higher than the saturated spin entropy $s / k_{B}=\ln (3)$, and even for adiabatic loading, a wider metallic transition region around the Mott shells is therefore expected because of the weaker Pomeranchuk cooling effect. The expected width is larger than the one observed in $\mathrm{SU}(6)$ and is consistent with the measured shape in the $\mathrm{SU}(3)$ case. We also observe that the entropy increase caused by the round-trip sequence is smaller in the $\mathrm{SU}(3)$ case, approximately $0.35 k_{B}$ as opposed to $0.6 k_{B}$ for $\mathrm{SU}(6)$. An interpretation could be that the freezing effect becomes more relevant for very low temperatures, where the resulting strong density gradients are expected to form in the atomic distribution.

An implication for the lattice loading procedure could be that mass flow is blocked before the density configuration of a fully equilibrated low-temperature gas is reached, at least in an ensemble with a strong Pomeranchuk effect. At the same time, this deviation from adiabaticity causes only moderate heating, with an entropy gain lower than what would correspond to a fully thermalized density distribution of the observed shape in the lattice. Understanding a possible edge-softening effect and its implications on the entropy distribution, as well as the underlying microscopic processes, could benefit from modeling the loading process itself, in analogy to current efforts for bosons in one dimension [29]. At the same time, the generalization to SU(6) 3D theory at low filling, and including spin correlations, is a challenge for current numerical methods.

In conclusion, we use in situ probing to measure the equation of state of an $\mathrm{SU}(N)$-symmetric Fermi gas in a 3D lattice. We obtain a very low compressibility in the Mott insulating phase and measure an entropy below that of uncorrelated spins. These findings make the system a promising starting point towards novel magnetically ordered many-body states in highly spin-symmetric systems $[4,5$, 11-13,27]. An incompressible phase with filling $n d^{3}=1$ is expected to faithfully realize the $\mathrm{SU}(N)$-symmetric Heisenberg model, paving the way towards studying unexplored systems with reduced dimensions such as chains [30-32] and new magnetic phases in square lattices [4].

We acknowledge fruitful discussions with Christophe Salomon and Tarik Yefsah. This work was supported by the ERC through the synergy grant UQUAM and by the European Union's Horizon 2020 funding (D.R.F.).

\section{APPENDIX A: PREPARATION OF SU $(N)$ FERMI GASES}

Approximately 3.5 million ground-state atoms of ${ }^{173} \mathrm{Yb}$, with equal populations in the nuclear spin states, are loaded into a crossed optical dipole trap operating at a wavelength of $\lambda=1064 \mathrm{~nm}$. Forced evaporative cooling produces a degenerate Fermi gas of 5000 atoms per spin state at temperature $T=0.07(1) T_{F}$, where $T_{F}$ is the Fermi temperature. The gas is very weakly interacting since $k_{F} a_{s} \lesssim 0.07$, with $a_{s}=199.4 a_{0}$ being the $s$-wave scattering length and $a_{0}$ the Bohr radius. We perform state preparation by driving the ${ }^{1} \mathrm{~S}_{0} \rightarrow{ }^{3} \mathrm{P}_{1}$ optical transition to remove unwanted nuclear spin states from the trap, in the presence of a homogeneous magnetic field that lifts the spin-state degeneracy. Using this technique, we generate an $\mathrm{SU}(3)$ Fermi gas with a temperature of $0.15(1) T_{F}$, with a residual fraction of unwanted spin components below 5\% per component.

\section{APPENDIX B: LOADING INTO THE OPTICAL LATTICE}

At the end of evaporation, the atoms are transferred into a cubic optical lattice in two steps. The sample is first loaded into a shallow lattice with depth $V=3 E_{r}$ in $150 \mathrm{~ms}$, avoiding band excitations. We then ramp the lattice depth to the final value, between $V=3 E_{r}$ and $V=15 E_{r}$, in $150 \mathrm{~ms}$. The atoms are trapped in the combined harmonic confinement produced by the lattice beams and the crossed optical dipole trap. During the ramp-up of the lattice, the power in the vertical arm of the dipole trap beam is ramped down for technical reasons. The resulting confinement frequencies vary between $\omega_{x, y, z}=2 \pi \times(31,42,183) \mathrm{Hz}$ at $3 E_{r}, \omega_{x, y, z}=2 \pi \times$ $(21,33,183) \mathrm{Hz}$ at $7 E_{r}$ and are approximately constant for $V>7 E_{r}$. We verified the validity of the harmonic approximation for our experimental trap configuration, by taking into account the combined Gaussian beam profiles. The variation of $U / t^{*}$ in the region occupied by the atoms is estimated to be below $8 \%$.

Dilute samples are loaded into the lattice in order to minimize losses and heating from three-body recombination, which is not suppressed by Pauli blocking for $N>2$ (see Appendix F). 


\section{APPENDIX C: HIGH-INTENSITY IMAGING}

The atom cloud has a typical optical density around 2 in the trap center. In order to perform in situ absorption imaging, we saturate the imaging transition ${ }^{1} \mathrm{~S}_{0} \rightarrow{ }^{1} \mathrm{P}_{1}$ with light intensity $I=15 I_{\text {sat }}$, where $I_{\text {sat }}=60 \mathrm{~mW} / \mathrm{cm}^{2}$. The optical resolution is about $1.2 \mu \mathrm{m}$, determined as described in Ref. [33]. The imaging pulse has a duration of $5 \mu \mathrm{s}$, sufficiently short to avoid atoms escaping from the focal plane and to minimize the Doppler shift due to photon scattering. Imaging is performed in the absence of magnetic fields in order to have spin-independent detection. Nevertheless, a $6 \%$ difference between the densities of the Mott plateaus is found by comparing the SU(6) and SU(3) gases, likely caused by the different line strengths within the hyperfine substructure of the imaging transition.

In order to correctly determine the density of the gas using high-intensity absorption imaging, we use the modified Lambert-Beer law [24], which accounts for saturation of the imaging transition:

$$
\mathrm{OD}=-\alpha \ln \left(\frac{I_{f}}{I_{i}}\right)+\frac{I_{i}-I_{f}}{I_{\mathrm{sat}}}
$$

where OD is the optical density, $I_{i}$ the incident light intensity, $I_{f}$ the final light intensity after absorption, and $I_{\text {sat }}$ the saturation intensity. The saturation intensity $I_{\text {sat }}$ is calculated from the linewidth and wavelength of the transition and additionally verified by an intensitydependent linewidth measurement.

The $\alpha$ parameter is extracted by varying the saturation parameter $I / I_{\text {sat }}$ and adjusting its value in a way that the measured optical density from Eq. (C1) is independent of the light intensity, as shown in Fig. 5. It accounts for a transition strength lower than 1 and, potentially, a systematic error when determining the value of the light intensity impinging on the atoms.

\section{APPENDIX D: ANALYTICAL MODELS}

In the absence of interactions, the EoS of an $N$-component harmonically confined Fermi gas is

$$
n(\mu, T)=-N\left(\frac{m k_{B} T}{2 \pi \hbar^{2}}\right)^{3 / 2} \operatorname{Li}_{3 / 2}\left(-e^{\mu / k_{B} T}\right),
$$

where $\mathrm{Li}_{3 / 2}$ denotes the polylogarithm of order $3 / 2$. This expression is also valid in the presence of a weak lattice potential, provided that the effective mass associated with the dispersion of the lowest band is used.

In the strongly interacting regime, the low-tunneling model is obtained using a high-temperature series expansion (HTSE) of the grand canonical potential up to second order in $t / k_{B} T[18,19,28]$ :
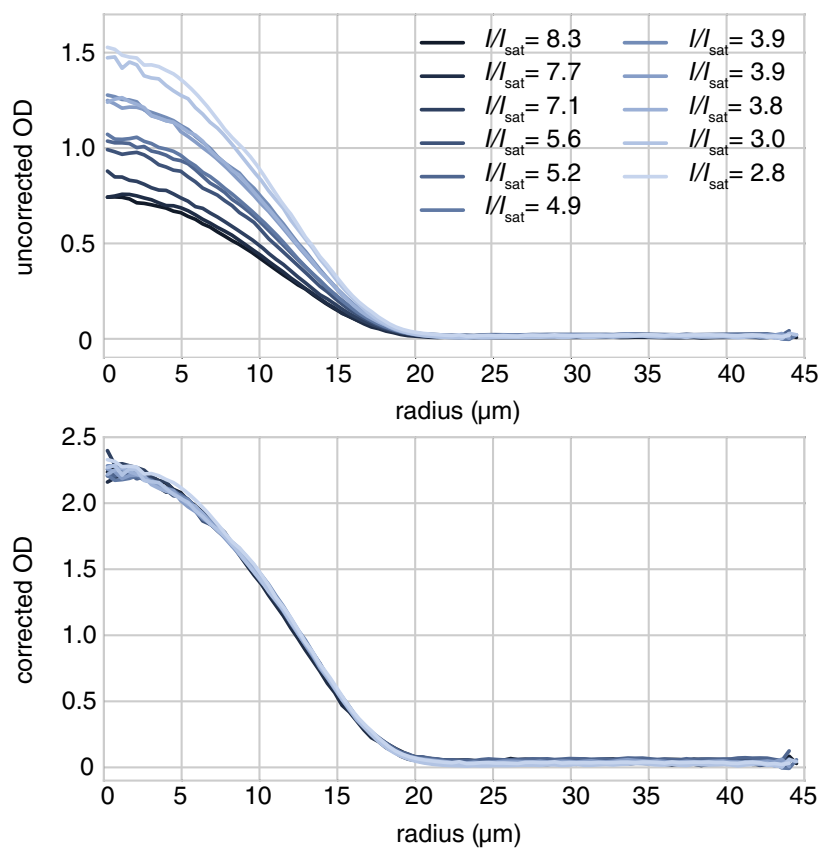

FIG. 5. Density profiles of the harmonically trapped SU(6) gas for various saturation parameters without saturation correction (top) and with saturation correction of Eq. (C1) with $\alpha=3.05$ (bottom).

$$
\begin{aligned}
\Omega= & \Omega_{0}+\Delta \Omega, \\
\Omega_{0}(\mu, T)= & -(1 / \beta) \ln Z_{0}(T, \mu), \\
Z_{0}(\mu, T)= & \sum_{n=0}^{N}\left(\begin{array}{c}
N \\
n
\end{array}\right) e^{-\beta[(U / 2) n(n-1)-\mu n]} \\
-\beta \Delta \Omega= & \left(\frac{\beta t}{Z_{0}}\right)^{2} z N\left[\frac{1}{2} \sum_{n_{1}=1}^{N}\left(\begin{array}{c}
N-1 \\
n_{1}-1
\end{array}\right)^{2} x^{2 n_{1}-1} y^{\left(n_{1}-1\right)^{2}}\right. \\
& -\frac{1}{\beta U} \sum_{n_{1} \neq n_{2}}^{N}\left(\begin{array}{c}
N-1 \\
n_{1}-1
\end{array}\right)\left(\begin{array}{l}
N-1 \\
n_{2}-1
\end{array}\right) \\
& \left.\times \frac{x^{n_{1}+n_{2}-1} y^{\frac{1}{2} n_{1}\left(n_{1}-1\right)+\frac{1}{2}\left(n_{2}-1\right)\left(n_{2}-2\right)}}{n_{1}-n_{2}}\right],
\end{aligned}
$$

with $z$ being the number of next neighbors in the lattice, $x=e^{\beta \mu}, y=e^{-\beta U}$, and $\Omega_{0}(\mu, T)$ the grand potential and $Z_{0}$ the partition function in the atomic limit.

\section{APPENDIX E: FITTING AND CALIBRATION}

The effective optical scattering cross section $\sigma$ of the atoms is calibrated using the known EoS of the polarized Fermi gas in the dipole trap with known trap frequencies. We obtain $\sigma / \sigma_{0}=0.222 \pm 0.034$ with $\sigma_{0}=(3 / 2 \pi) \lambda^{2}$.

The HTSE model fit to the measured EoS, effectively fitting the amplitude of the plateau, is more sensitive to the optical cross section and yields $\sigma / \sigma_{0}=0.257 \pm 0.013$, which is used in this work. The plateau density for the $N=6$ data shown is therefore fixed to $1 / d^{3}$, whereas an independent fit for the $N=3$ case yields $n d^{3}=0.94 \pm 0.02$, 
possibly due to residual spin dependence of the imaging technique.

\section{APPENDIX F: CHARACTERIZATION OF THREE-BODY LOSSES}

In order to avoid three-body losses in the lattice, we keep the central average filling below $n d^{3} \simeq 1.7$. For higher filling factors, we observe a fast density decay in the central region of the cloud in addition to a slow density decay due to vacuum losses and technical heating of the lattice beams, as shown in Fig. 6. We fit a double exponential decay to the core density of the cloud

$$
n(t)=n_{d} * e^{-t / \tau_{v}}+n_{3} * e^{-t\left(3 / \tau_{v}+1 / \tau_{3}\right)},
$$

where $n_{d}$ is the density of singly and doubly occupied sites, $n_{3}$ is the density of triply occupied sites, and $\tau_{v}, \tau_{3}$ denote the vacuum lifetime and three-body loss time scale, respectively. We extract a loss rate $\gamma=1 / \tau_{3}=2.4(3) \mathrm{Hz}$ in a $15 E_{r}$ deep lattice. The scaling of the loss rate as a function of the lattice depth is found to be compatible with the expected scaling of a three-body decay rate,

$$
\gamma=\beta_{3} \int d^{3} \mathbf{x} w(\mathbf{x})^{6},
$$

where $w(\mathbf{x})$ is the lattice depth-dependent Wannier function $[34,35]$. Using Eq. (F2), we determine the $\beta_{3}$ coefficient independently for three lattice depths $V=15 E_{r}, V=22 E_{r}$, and $V=30 E_{r}$. From these measurements, we obtain an estimate of the three-body loss rate coefficient $\beta_{3}=$ $2.3(6) \times 10^{-29} \mathrm{~cm}^{6} / \mathrm{s}$. This value is of the same order of magnitude as previously reported three-body loss rate coefficients in alkali atoms [34].

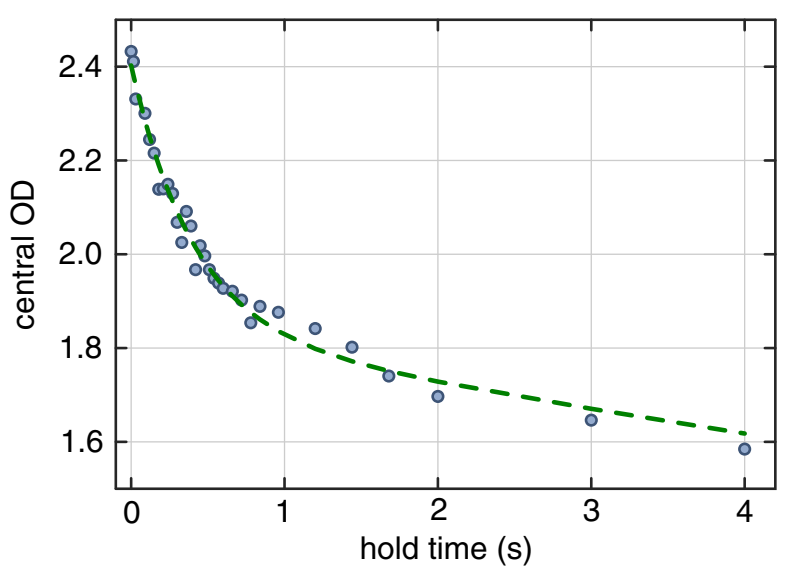

FIG. 6. Atom loss at high densities in the center of the cloud in $V=15 E_{r}$ deep lattice (circles). The fit function (dashed line) is a double exponential decay described by Eq. (F1).
[1] P. W. Anderson, The Resonating Valence Bond State in $\mathrm{La}_{2} \mathrm{CuO}_{4}$ and Superconductivity, Science 235, 1196 (1987).

[2] M. Imada, A. Fujimori, and Y. Tokura, Metal-Insulator Transitions, Rev. Mod. Phys. 70, 1039 (1998).

[3] Y. Tokura and N. Nagaosa, Orbital Physics in TransitionMetal Oxides, Science 288, 462 (2000).

[4] C. Honerkamp and W. Hofstetter, Ultracold Fermions and the $\mathrm{SU}(N)$ Hubbard Model, Phys. Rev. Lett. 92, 170403 (2004).

[5] R. W. Cherng, G. Refael, and E. Demler, Superfluidity and Magnetism in Multicomponent Ultracold Fermions, Phys. Rev. Lett. 99, 130406 (2007).

[6] M. Hermele, V. Gurarie, and A. M. Rey, Mott Insulators of Ultracold Fermionic Alkaline Earth Atoms: Underconstrained Magnetism and Chiral Spin Liquid, Phys. Rev. Lett. 103, 135301 (2009).

[7] A. V. Gorshkov, M. Hermele, V. Gurarie, C. Xu, P. S. Julienne, J. Ye, P. Zoller, E. Demler, M. D. Lukin, and A. M. Rey, Two-Orbital SU(N) Magnetism with Ultracold Alkaline-Earth Atoms, Nat. Phys. 6, 289 (2010).

[8] A. Rapp and A. Rosch, Ground-State Phase Diagram of the Repulsive SU(3) Hubbard Model in the Gutzwiller Approximation, Phys. Rev. A 83, 053605 (2011).

[9] K. Inaba and S.-I. Suga, Superfluid, Staggered State, and Mott Insulator of Repulsively Interacting Three-Component Fermionic Atoms in Optical Lattices, Mod. Phys. Lett. B 27, 1330008 (2013).

[10] H. Song and M. Hermele, Mott Insulators of Ultracold Fermionic Alkaline Earth Atoms in Three Dimensions, Phys. Rev. B 87, 144423 (2013).

[11] M. A. Cazalilla and A. M. Rey, Ultracold Fermi Gases with Emergent SU(N) Symmetry, Rep. Prog. Phys. 77, 124401 (2014).

[12] A. Sotnikov and W. Hofstetter, Magnetic Ordering of ThreeComponent Ultracold Fermionic Mixtures in Optical Lattices, Phys. Rev. A 89, 063601 (2014).

[13] A. Sotnikov, Critical Entropies and Magnetic-PhaseDiagram Analysis of Ultracold Three-Component Fermionic Mixtures in Optical Lattices, Phys. Rev. A 92, 023633 (2015).

[14] T. Esslinger, Fermi-Hubbard Physics with Atoms in an Optical Lattice, Annu. Rev. Condens. Matter Phys. 1, 129 (2010).

[15] B. J. DeSalvo, M. Yan, P. G. Mickelson, Y. N. Martinez de Escobar, and T. C. Killian, Degenerate Fermi Gas of ${ }^{87} \mathrm{Sr}$, Phys. Rev. Lett. 105, 030402 (2010).

[16] T. Fukuhara, Y. Takasu, M. Kumakura, and Y. Takahashi, Degenerate Fermi Gases of Ytterbium, Phys. Rev. Lett. 98, 030401 (2007).

[17] F. Scazza, C. Hofrichter, M. Höfer, P. C. De Groot, I. Bloch, and S. Fölling, Observation of Two-Orbital Spin-Exchange Interactions with Ultracold SU(N)-Symmetric Fermions, Nat. Phys. 10, 779 (2014).

[18] K. R. A. Hazzard, V. Gurarie, M. Hermele, and A. M. Rey, High-Temperature Properties of Fermionic Alkaline-EarthMetal Atoms in Optical Lattices, Phys. Rev. A 85, 041604 (2012).

[19] S. Taie, R. Yamazaki, S. Sugawa, and Y. Takahashi, An SU(6) Mott Insulator of an Atomic Fermi Gas Realized by 
Large-Spin Pomeranchuk Cooling, Nat. Phys. 8, 825 (2012).

[20] S. Nascimbène, N. Navon, K. J. Jiang, F. Chevy, and C. Salomon, Exploring the Thermodynamics of a Universal Fermi Gas, Nature (London) 463, 1057 (2010).

[21] K. Van Houcke, F. Werner, E. Kozik, N. Prokof'ev, B. Svistunov, M. J. H. Ku, A. T. Sommer, L. W. Cheuk, A. Schirotzek, and M. W. Zwierlein, Feynman diagrams versus Fermi-gas Feynman emulator, Nat. Phys. 8, 366 (2012).

[22] R. W. Helmes, T. A. Costi, and A. Rosch, Mott Transition of Fermionic Atoms in a Three-Dimensional Optical Trap, Phys. Rev. Lett. 100, 056403 (2008).

[23] E. V. Gorelik and N. Blümer, Mott Transitions in Ternary Flavor Mixtures of Ultracold Fermions on Optical Lattices, Phys. Rev. A 80, 051602 (2009).

[24] G. Reinaudi, T. Lahaye, Z. Wang, and D. Guéry-Odelin, Strong Saturation Absorption Imaging of Dense Clouds of Ultracold Atoms, Opt. Lett. 32, 3143 (2007).

[25] V. Dribinski, A. Ossadtchi, V. A. Mandelshtam, and H. Reisler, Reconstruction of Abel-Transformable Images: The Gaussian Basis-Set Expansion Abel Transform Method, Rev. Sci. Instrum. 73, 2634 (2002).

[26] P. M. Duarte, R. A. Hart, T.-L. Yang, X. Liu, T. Paiva, E. Khatami, R. T. Scalettar, N. Trivedi, and R. G. Hulet, Compressibility of a Fermionic Mott Insulator of Ultracold Atoms, Phys. Rev. Lett. 114, 070403 (2015).

[27] S.-K. Yip, B.-L. Huang, and J.-S. Kao, Theory of $\mathrm{SU}(N)$ Fermi Liquids, Phys. Rev. A 89, 043610 (2014).
[28] J. Oitmaa, C. Hamer, and W. Zheng, Series Expansion Methods for Strongly Interacting Lattice Models (Cambridge University Press, Cambridge, England, 2010).

[29] M. Dolfi, A. Kantian, B. Bauer, and M. Troyer, Minimizing Nonadiabaticities in Optical-Lattice Loading, Phys. Rev. A 91, 033407 (2015).

[30] S. R. Manmana, K. R. A. Hazzard, G. Chen, A. E. Feiguin, and A. M. Rey, SU(N) Magnetism in Chains of Ultracold Alkaline-Earth-Metal Atoms: Mott Transitions and Quantum Correlations, Phys. Rev. A 84, 043601 (2011).

[31] L. Messio and F. Mila, Entropy Dependence of Correlations in One-Dimensional SU(N) Antiferromagnets, Phys. Rev. Lett. 109, 205306 (2012).

[32] S. Capponi, P. Lecheminant, and K. Totsuka, Phases of One-Dimensional SU(N) Cold Atomic Fermi Gases-From Molecular Luttinger Liquids to Topological Phases, Ann. Phys. (Amsterdam) 367, 50 (2016).

[33] C.-L. Hung, X. Zhang, L.-C. Ha, S.-K. Tung, N. Gemelke, and C. Chin, Extracting Density-Density Correlations from In Situ Images of Atomic Quantum Gases, New J. Phys. 13, 075019 (2011).

[34] B. D. Esry, C. H. Greene, and J. P. Burke, Recombination of Three Atoms in the Ultracold Limit, Phys. Rev. Lett. 83, 1751 (1999).

[35] M. W. Jack and M. Yamashita, Signatures of the Quantum Fluctuations of Cold Atoms in an Optical Lattice in the Three-Body Loss Rate, Phys. Rev. A 67, 033605 (2003). 